\title{
ON HOLOMORPHICALLY PROJECTIVE MAPPINGS OF PARABOLIC KÄHLER MANIFOLDS
}

\author{
P. PEŠKA, J. MIKEŠS, H. CHUDÁ, AND M. SHIHA
}

Received 30 November, 2015

\begin{abstract}
In this paper we study fundamental equations of holomorphically projective mappings of parabolic Kähler spaces (which are generalized classical, pseudo- and hyperbolic Kähler spaces) with respect to the smoothness class of metrics. We show that holomorphically projective mappings preserve the smoothness class of metrics.
\end{abstract}

2010 Mathematics Subject Classification: 53B20; 53B21; 53B30; 53C26

Keywords: holomorphically projective mappings, smoothness class, parabolic Kähler manifold

\section{INTRODUCTION}

First we study the general dependence of holomorphically-projective mappings of parabolic Kähler manifolds in dependence on the smoothness class of the metric. We present well known facts, which were proved by M. Shiha, J. Mikeš et al, see $[2,3,14,17-21]$.

I. Hinterleitner [5] has solved the analogically problems for classical, pseudo- and hyperbolic Kähler manifolds. In this paper were clarified results which were proved by Bácsó, Domashev, Kurbatova, Mikeš, Prvanović, Otsuki, Tashiro, see [4, 10-13, $15,16,23,25]$. In these results no details about the smoothness class of the metric were stressed. They were formulated "for sufficiently smooth" geometric objects. This result was inspired in [6,7] of geodesic mappings.

\section{PARABOLIC KäHLER MANIFOLDS}

In the following definition we introduce generalizations of Kähler manifolds [8], see $[9,11,13]$. A basis on this definition see the monography by V.V. Vishnevskii, A.P. Shirokov and V.V. Shurigin [24].

Definition 1. An $n$-dimensional (pseudo-) Riemannian manifold $(M, g)$ is called an $m$-parabolic Kähler manifold $K_{n}^{o(m)}$, if beside the metric tensor $g$, a tensor field

The paper was supported by the project IGA PrF 2014016 Palacký University Olomouc. 
$F$ of a rank $m \geq 2$ of type $(1,1)$ is given on the manifold $M_{n}$, called a structure $F$, such that the following conditions hold:

$$
F^{2}=0, \quad g(X, F X)=0, \quad \nabla F=0,
$$

where $X$ is an arbitrary vector of $T M_{n}$, and $\nabla$ denotes the covariant derivative in $K_{n}^{o(m)}$.

We remind, that Kähler spaces, were characterized by conditions $F^{2}=-I d$, $g(X, F X)=0, \nabla F=0$, were first considered by P.A. Shirokov, see [22]. Independently they were studied by E. Kähler [8]. Hyperbolic Kähler space (also para Kähler space, see D.V. Alekseevsky [1]) characterized $F^{2}=I d, g(X, F X)=0, \nabla F=0$, were considered P.K. Rashevskij, see [9].

\section{HOLOMORPHICALLY-PROJECTIVE MAPPING THEORY FOR $K_{n}^{o(m)} \rightarrow \bar{K}_{n}^{o(\bar{m})}$ OF CLASS $C^{1}$}

Assume the parabolic Kähler manifolds $K_{n}^{o(m)}=(M, g, F)$ and $\bar{K}_{n}^{o(\bar{m})}=$ $(\bar{M}, \bar{g}, \bar{F})$ with metrics $g$ and $\bar{g}$, structures $F$ and $\bar{F}$, Levi-Civita connections $\nabla$ and $\bar{\nabla}$, respectively. Here $\bar{K}_{n}, \bar{K}_{n} \in C^{1}$, i.e. $g, \bar{g} \in C^{1}$ which means that their components $g_{i j}, \bar{g}_{i j} \in C^{1}$. Likewise, as in $[17,18]$ we introduce the following notations, this is an analogy by [15], see [11, p. 240].

Definition 2. A curve $\ell$ in $K_{n}$ which is given by the equation $\ell=\ell(t), \lambda=$ $d \ell / d t(\neq 0), t \in I$, where $t$ is a parameter is called analytical planar, if under the parallel translation along the curve, the tangent vector $\lambda$ belongs to the twodimensional distribution $D=\operatorname{Span}\{\lambda, F \lambda\}$ generated by $\lambda$ and its conjugate $F \lambda$, that is, it satisfies

$$
\nabla_{t} \lambda=a(t) \lambda+b(\lambda) F \lambda
$$

where $a(t)$ and $b(t)$ are some functions of the parameter $t$. Particularly, in the case $b(t)=0$, an analytical planar curve is a geodesic.

Definition 3. A diffeomorphism $f: K_{n}^{o(m)} \rightarrow \bar{K}_{n}^{o(\bar{m})}$ is called a holomorphicallyprojective mapping of $K_{n}^{o(m)}$ onto $\bar{K}_{n}^{o(\bar{m})}$ if $f$ maps any analytical planar curve in $K_{n}^{o(m)}$ onto an analytical planar curve in $\bar{K}_{n}^{o(\bar{m})}$.

Assume a holomorphically-projective mapping $f: K_{n}^{o(m)} \rightarrow \bar{K}_{n}^{o(\bar{m})}$. Since $f$ is a diffeomorphism, we can suppose local coordinate charts on $M$ or $\bar{M}$, respectively, such that locally, $f: K_{n}^{o(m)} \rightarrow \bar{K}_{n}^{o(\bar{m})}$ maps points onto points with the same coordinates, and $\bar{M}=M$. A manifold $K_{n}^{o(m)}$ admits a holomorphically-projective mapping onto $\bar{K}_{n}^{o(\bar{m})}$ if and only if the following equations [17]:

$$
\bar{\nabla}_{X} Y=\nabla_{X} Y+\psi(X) Y+\psi(Y) X+\varphi(F X) F Y+\varphi(F Y) F X
$$

hold for any tangent fields $X, Y$ and where $\psi$ is a gradient-like form and $\psi(X)=$ $\varphi(F X)$. If $\psi \equiv 0$ than $f$ is affine or trivially holomorphically-projective . Moreover, 
structures $F$ and $\bar{F}$ are preserved, i.e. $\bar{F}=F$, and $\bar{m}=m$. This fact implies from the theory of $F$-planar mappings, see [11, pp. 219-220]. In local form:

$$
\bar{\Gamma}_{i j}^{h}=\Gamma_{i j}^{h}+\psi_{i} \delta_{j}^{h}+\psi_{j} \delta_{i}^{h}+\varphi_{i} F_{j}^{h}+\varphi_{j} F_{i}^{h}, \quad \psi_{i}=\varphi_{j} F_{i}^{j},
$$

where $\Gamma_{i j}^{h}$ and $\bar{\Gamma}_{i j}^{h}$ are the Christoffel symbols of $K_{n}$ and $\bar{K}_{n}, \psi_{i}, F_{i}^{h}$ are components of $\psi, F$ and $\delta_{i}^{h}$ is the Kronecker delta,

$$
\psi_{i}=\frac{\partial \Psi}{\partial x^{i}}, \quad \Psi=\frac{1}{2(n+2)} \ln \left|\frac{\operatorname{det} \bar{g}}{\operatorname{det} g}\right| .
$$

Here and in the following we will use the conjugation operation of indices in the way

$$
A_{\ldots \bar{i} \ldots}=A_{\ldots k} \ldots F_{i}^{k} .
$$

Equations (3.1) are equivalent to the following equations

$$
\begin{aligned}
\nabla_{Z} \bar{g}(X, Y)= & 2 \psi(Z) \bar{g}(X, Y)+\psi(X) \bar{g}(Y, Z)+\psi(Y) \bar{g}(X, Z) \\
& +\varphi(F X) \bar{g}(F Y, Z)+\varphi(F Y) \bar{g}(F X, Z) .
\end{aligned}
$$

In local form:

$$
\bar{g}_{i j, k}=2 \psi_{k} \bar{g}_{i j}+\psi_{i} \bar{g}_{j k}+\psi \bar{g}_{i k}+\varphi_{i} \bar{g}_{\bar{j} k}+\varphi_{j} \bar{g}_{\bar{i} k},
$$

where "," denotes the covariant derivative on $K_{n}^{o(m)}$. M. Shiha and J. Mikeš [18] proved that equations (3.1) and (3.2) are equivalent to

$$
\nabla_{Z} a(X, Y)=\lambda(X) g(Y, Z)+\lambda(Y) g(X, Z)+\theta(X) g(F Y, Z)+\theta(Y) g(F X, Z) .
$$

In local form:

$$
a_{i j, k}=\lambda_{i} g_{j k}+\lambda_{j} g_{i k}+\theta_{i} g_{\bar{j} k}+\theta_{j} g_{\bar{i} k}
$$

where
(a) $a_{i j}=\mathrm{e}^{2 \Psi} \bar{g}^{\alpha \beta} g_{\alpha i} g_{\beta j}$,
(b) $\lambda_{i}=-\mathrm{e}^{2 \Psi} \bar{g}^{\alpha \beta} g_{\beta i} \psi_{\alpha}$,
(c) $\theta_{i}=-\mathrm{e}^{2 \Psi} \bar{g}^{\alpha \beta} g_{\beta i} \varphi_{\alpha}$.

From (3.3) follows that $\lambda_{i}$ is gradient-like vector and it holds

$$
\lambda_{i}=\partial_{i} \Lambda, \quad \Lambda=1 / 4 a_{\alpha \beta} g^{\alpha \beta} .
$$

On the other hand [11]:

$$
\bar{g}_{i j}=\mathrm{e}^{2 \Psi} \tilde{g}_{i j}, \quad \Psi=\frac{1}{2} \ln \left|\frac{\operatorname{det} \tilde{g}}{\operatorname{det} g}\right|, \quad\left\|\tilde{g}_{i j}\right\|=\left\|g^{i \alpha} g^{j \beta} a_{\alpha \beta}\right\|^{-1} .
$$

The above formulas are the criterion for holomorphically-projective mappings $K_{n}^{o(m)} \rightarrow \bar{K}_{n}^{o(m)}$, globally as well as locally. 
Theorem 1. A diffeomorphism $f: K_{n}^{o(m)} \rightarrow \bar{K}_{n}^{o(\bar{m})}$ is a holomorphicallyprojective mapping if and only if there exist a solution of the following linear Cauchylike system

$$
\begin{aligned}
\text { a) } a_{i j, k} & =\lambda_{(i} g_{j) k}+\theta_{(i} F_{j) k} ; \\
\text { b) } \theta_{i, j} & =\tau F_{i j}+a_{\alpha \beta} M_{1 \mid i j}^{\alpha \beta} ; \\
\text { c) } \tau_{, i} & =\theta_{\alpha} M_{2 \mid i}^{\alpha}+a_{\alpha \beta} M_{3 \mid i}^{\alpha \beta}
\end{aligned}
$$

on unknown tensor $a_{i j}\left(a_{i j}=a_{j i}, a_{\bar{i} j}+a_{i \bar{j}}=0, \operatorname{det} a_{i j} \neq 0\right)$, a vector $\lambda_{i}$, and $a$ function $\tau$. Here $M_{1 \mid i j}^{\alpha \beta}, M_{2 \mid i}^{\alpha}, M_{3 \mid i}^{\alpha \beta}$ are tensors determined from metric and structure tensors $g_{i j}$ and $F_{i}^{h}$ of the space $K_{n}^{o(m)}$.

Remark 1. This theorem was proved with assuming that $K_{n}^{o(m)}$ and $\bar{K}_{n}^{o(m)}$ belong to $C^{3}$ class. We will prove, that the Theorem 1 valides too if $K_{n}^{o(m)} \in C^{3}$ and $\bar{K}_{n}^{o(\bar{m})} \in C^{2}$.

The system (3.7) has at most one solution for the initial values in a point $x_{0}$ : $a_{i j}\left(x_{0}\right), \lambda_{i}\left(x_{0}\right)$ and $\tau\left(x_{0}\right)$. Hence, the general solution of this system depends on no more than $(n+2)(n+1) / 2-m(n-m+1)$ essential parameters.

The integrability of conditions (3.7) and their differential prolongations are linear algebraic equations on the components of the unknown tensors $a_{i j}, \lambda_{i j}$ and $\tau$ with coefficients from $K_{n}^{o(m)}$.

\section{HOLOMORPHICALLY-PROJECTIVE MAPPING OF PARABOLIC KÄHLER SPACE OF CLASS $C^{2}$}

The direct substitution of (3.1) implies that Riemannian tensors of spaces $K_{n}^{o(m)}$ and $\bar{K}_{n}^{o(m)}$, which are holomorphically-projectively corresponding of $m$-parabolic Kähler space are connected by the following relations:

$$
\bar{R}_{i j k}^{h}=R_{i j k}^{h}+\delta_{k}^{h} \psi_{i j}-\delta_{j}^{h} \psi_{i k}+F_{k}^{h} \varphi_{i j}-F_{j}^{h} \varphi_{i k}+F_{i}^{h} \varphi_{[k j]},
$$

where $\bar{R}_{i j k}^{h}, R_{i j k}^{h}$ are Riemannian tensors of $K_{n}^{o(m)}$ and $\bar{K}_{n}^{o(m)}$,

$$
\varphi_{i k} \equiv \varphi_{i, j}-\psi_{i} \varphi_{j}-\varphi_{i} \psi_{j} ; \quad \psi_{i j} \equiv \varphi_{\bar{i} j}
$$

Tensor $\bar{\varphi}_{i j}$ has this form:

$$
\psi_{i j}=\psi_{i, j}-\psi_{i} \psi_{j}
$$

As $\psi_{i}$ is a gradient, that this tensor is symmetrical, that is

$$
\psi_{i j}=\psi_{j i} .
$$

Contracting (4.1) with respect to indices $h$ and $k$, we obtain connection between Ricci tensors

$$
\bar{R}_{i j}=R_{i j}+n \psi_{i j}
$$


where $R_{i j}$ and $\bar{R}_{i j}$ are Ricci tensors of $K_{n}^{o(m)}$ and $\bar{K}_{n}^{o(m)}$.

Next, we will proceed similarly as in work of M. Shiha [17,18]. Let we consider the integrability condition of equation (3.3):

$$
a_{\alpha(i} R_{j) k l}^{\alpha}=g_{k(i} \lambda_{j), l}-g_{l(} \lambda_{j), k}-F_{k(i} \theta_{j), l}+F_{l(i} \theta_{j), k} .
$$

Contracting (4.6) with $F_{k^{\prime}}^{k}$ and also with $F_{l^{\prime}}^{l}$, we obtain two expressions. After removing primes, we sum them up. Since $K_{n}^{o(m)}$ it holds $R_{i \alpha k}^{h}+R_{i j \alpha}^{h} F_{k}^{\alpha}=0$, we get $F_{k(i} \Lambda_{j) l}-F_{l(i} \Lambda_{j) k}=0$, where $\Lambda_{i j} \equiv \lambda_{i, j}+\theta_{i, \alpha} F_{j}^{\alpha}$. It follows that $\Lambda_{i j}=\Lambda F_{i j}$, i.e. $\lambda_{i, j}+\theta_{i, \alpha} F_{j}^{\alpha}=\Lambda F_{i j}$ where $\Lambda$ is a function. Contracting (4.6) with $g^{j k}$, we obtain

$$
n \lambda_{i, j}=\mu g_{i j}+v F_{i j}-a_{\alpha i} R_{j}^{\alpha}-a_{\alpha \beta} R_{. i j .}^{\alpha . \beta} .
$$

where $R_{i j k}^{h}$ and $R_{i j}$ are Riemann and Ricci tensors, respectively, the operation of lifting and lowering indices are induced by the metric tensor, and $\mu, v$ are certain functions. After symmetrizing (4.7) we get

$$
n \lambda_{i, j}=\mu g_{i j}-\frac{1}{2} a_{\alpha(i} R_{j)}^{\alpha}-a_{\alpha \beta} R_{. i j .}^{\alpha . . \beta} .
$$

Substituting (4.8) to (4.6) we obtain

$$
a_{\alpha \beta} M_{j k l}^{\alpha \beta}=F_{l i} \theta_{j, k}+F_{l j} \theta_{i, k}-F_{k i} \theta_{j, l}+F_{k j} \theta_{i, l} .
$$

From this implies that $M$ are tensors determined by $g_{i j}$ and $F_{i}^{h}$ on $K_{n}^{o(m)}$. More precisely

$$
M_{i j k l}^{\alpha \beta} \equiv \delta_{(i}^{\alpha} R_{j) k l}^{\beta}+M_{4 \mid k(i}^{\alpha \beta} g_{j) l}-M_{4 \mid l(i}^{\alpha \beta} g_{j) k} ; \quad n M_{4 \mid l i}^{\alpha \beta} \equiv \frac{1}{2} \delta_{(i}^{\alpha} R_{j)}^{\beta}-R_{. i j .}^{\alpha . . \beta},
$$

where $\delta_{i}^{h}$ is the Kronecker symbol. Let $\varepsilon^{j}$ and $v^{k}$ be vectors such that $\varepsilon^{j} v^{k} F_{j k}=1$. Denote $M_{i} \equiv \varepsilon^{\alpha} F_{\alpha i}$. Contracting (4.9) with $\varepsilon^{i} \varepsilon^{j} v^{k}$ we get

$$
\varepsilon^{\alpha} \theta_{\alpha, l}=\tau M_{l}+a_{\alpha \beta} M_{5 \mid l}^{\alpha \beta},
$$

where $\tau \equiv \lambda_{\alpha, \beta} \varepsilon^{\alpha} \nu^{\beta}$. Contracting (4.9) with $\varepsilon^{j} v^{k}$ and using (4.10) we have the following formula

$$
\theta_{i, j}=\tau F_{i j}+\Lambda_{i} M_{j}+a_{\alpha \beta} M_{6 \mid i j}^{\alpha \beta},
$$

where $\lambda_{i}$ is a vector. Substituting (4.11) into (4.9) we have

$$
\Lambda_{i}\left(M_{k} F_{l j}-M_{l} F_{k j}\right)+\Lambda_{j}\left(M_{k} F_{l i}-M_{l} F_{k i}\right)=a_{\alpha \beta} M_{7 \mid i j k l}^{\alpha \beta} .
$$

So we proved the following lemma:

Lemma 1. Let $K_{n}^{o(m)}$ and $\bar{K}_{n}^{o(\bar{m})}$ belong to class $C^{2}$. If $K_{n}^{o(m)}$ admit a holomorphically-projective mapping onto $\bar{K}_{n}^{o(\bar{m})}$ than formulae (3.7a) and (3.7b) hold. 
5. HOLOMORPHICALLY-PROJECTIVE MAPPING $K_{n}^{o(m)} \rightarrow \bar{K}_{n}^{o(\bar{m})}$

$$
\text { FOR } K_{n}^{o(m)} \in C^{r}(r \geq 3) \text { AND } \bar{K}_{n}^{o(\bar{m})} \in C^{2}
$$

Theorem 2. Let $K_{n}^{o(m)} \in C^{r}(r \geq 3)$ and $\bar{K}_{n}^{o(\bar{m})} \in C^{2}$. If $K_{n}^{o(m)}$ admits holomorphically-projective mapping onto $\bar{K}_{n}^{o(\bar{m})}$ then $\bar{K}_{n}^{o(\bar{m})} \in C^{r}$.

Proof of Theorem 2 is based on the proof of Theorem 3.

Theorem 3. Let $K_{n}^{o(m)} \in C^{3}$ and $\bar{K}_{n}^{o(\bar{m})} \in C^{2}$. If $K_{n}^{o(m)}$ admits holomorphicallyprojective mapping onto $\bar{K}_{n}^{o(\bar{m})}$ then $\bar{K}_{n}^{o(\bar{m})} \in C^{3}$.

For first, we prove that metric $g$ and structure $F$ have the same differentiation.

Lemma 2. If $K_{n}^{o(m)}=(M, g, F) \in C^{r}$, i.e. $g(x) \in C^{r}$, then $F(x) \in C^{r}$, for $r \in \mathbb{N}$ and $r=\infty, \omega$.

Proof. Let $K_{n}^{o(m)} \in C^{r}$, i.e. the components of metric $g_{i j}(x) \in C^{r}$ in a coordinate chart $x$. It is a priori valid, that $F_{i}^{h} \in C^{1}$. The formula $\nabla F=0$ can be written $\partial_{k} F_{i}^{h}=F_{\alpha}^{h} \Gamma_{i k}^{\alpha}-F_{i}^{\alpha} \Gamma_{\alpha k}^{h}$, where $\Gamma_{i j k}=1 / 2\left(\partial_{i} g_{j k}+\partial_{j} g_{i k}-\partial_{k} g_{i j}\right), \partial_{k}=\partial / \partial x^{k}$, and $\Gamma_{i j}^{h}=g^{h k} \Gamma_{i j k}$ are the Christoffel symbols of the first and second kind, respectively. It holds, that $\Gamma_{i j k}$ and $\Gamma_{i j}^{h} \in C^{r-1}$. From this equation immediately follows $F_{i}^{h}(x) \in C^{r}$, i.e. $F \in C^{r}$.

For proving Theorem 3, we need the lemmae:

Lemma 3 ([9]). Let $\lambda^{h}(x) \in C^{1}$ be a vector field, $\Phi_{i}^{h}=\left(\begin{array}{l|l}I & 0 \\ \hline 0 & 0\end{array}\right)$ is special form with $I=\delta_{b}^{a}$, where $1 \leq a, b \leq r, r \geq 2$. If $\partial_{i} \lambda^{h}-\rho \Phi_{i}^{h} \in C^{1}$ then $\lambda^{h} \in C^{2}$ and $\rho \in C^{1}$.

Lemma 4. Let $\lambda^{h}(x) \in C^{1}$ be a vector field, $F_{i}^{h}(x) \in C^{2}$ is a tensor field of rank $F \geq 2$. If $\partial_{i} \lambda^{h}-\rho F_{i}^{h} \in C^{1}$ then $\lambda^{h} \in C^{2}$ and $\rho \in C^{1}$.

Proof. Let

$$
\partial_{i} \lambda^{h}-\rho F_{i}^{h}(x)=f_{i}^{h}(x) \in C^{1} .
$$

Because rank $F_{i}^{h} \geq r$ and $F_{i}^{h}(x) \in C^{2}$, then exist a regular tensor field $\Omega_{i}^{h}(x) \in C^{2}$ that $F_{i}^{h} \Omega_{j}^{i}=\Phi_{i}^{h}$

We put $v^{h}=\lambda^{\alpha} \Omega_{\alpha}^{h}$. Then we have

$$
\partial_{i} v^{h}-\rho \Phi_{\alpha}^{h}=\partial_{i}\left(\lambda^{\alpha} \Omega_{\alpha}^{h}\right)-\rho \Phi_{\alpha}^{h}=f_{i}^{\alpha} \Omega_{\alpha}^{h}+\lambda^{\alpha} \partial_{i} \Omega_{\alpha}^{h} .
$$

Because $f_{i}^{\alpha} \Omega_{\alpha}^{h}+\lambda^{\alpha} \partial_{i} \Omega_{\alpha}^{h} \in C^{1}$, from Lemma 3 it implies $v^{h} \in C^{2}$ and $\rho \in C^{1}$. Since $\rho \in C^{1}$, then $\lambda^{h}(x) \in C^{2}$.

It follows prooving of Theorem 3: 
Proof. Equations (3.7b) can be written in the following form:

$$
\partial_{j} \theta^{h}=\tau F_{j}^{h}-\theta^{\alpha} \Gamma_{\alpha j}^{h}+a_{\alpha \beta} M_{1 \mid j}^{\alpha \beta h},
$$

i.e. $\partial_{j} \theta^{h}-\tau F_{j}^{h}=f_{j}^{h}$, where $f_{j}^{h}=-\theta^{\alpha} \Gamma_{\alpha j}^{h}+a_{\alpha \beta} M_{1 \mid j}^{\alpha \beta h}$. Because $f_{j}^{i} \in C^{1}$ and $F_{i}^{h} \in C^{3}$ then from Lemma 4 follows $\theta^{i} \in C^{2}$ and $\tau \in C^{1}$. Using (3.7a) and (3.7b) we obtain $a_{i j}(x) \in C^{3}$ and $\Psi \in C^{3}$. Finally, from (3.6) we have $\bar{g}_{i j}(x) \in C^{3}$.

In to this moment, we proved formulae (3.7a) and (3.7b). For complete proof of Theorem 1, we have to prove the latest formula $(3.7 \mathrm{c})$.

Proof. Proof of Theorem 3 allows us differentiation of equation (3.7b). Application to this, we have:

$$
\theta_{i, j k}=\tau_{, k} F_{i j}+a_{\alpha \beta, k} M_{1 \mid i j}^{\alpha \beta}+a_{\alpha \beta} M_{1 \mid i j, k}^{\alpha \beta} .
$$

After alternation with respect to indices $j$ and $k$ and using Ricci identity, we have

$$
\tau_{, j} F_{i k}-\tau_{, k} F_{i j}=a_{\alpha \beta} M_{8 \mid i j k}^{\alpha \beta}+\theta_{\alpha} M_{9 \mid i j k}^{\alpha \beta} .
$$

Contracting (5.2) with $\varepsilon^{i} \varepsilon^{j} v^{k}$, we obtain $\tau_{, a} \varepsilon^{\alpha}=a_{\alpha \beta} M_{9 \mid}^{\alpha \beta}+\lambda_{\alpha} M_{10}^{\alpha \beta}$. Finally, contracting (5.2) with $\varepsilon^{j} v^{i}$, we have (3.7c) and Theorem 1 is proved.

At the end, we prove Theorem 2.

Proof. If $K_{n}^{o(m)} \in C^{r}(r \geq 3)$ and $\bar{K}_{n}^{o(\bar{m})} \in C^{2}$, then by Theorem 3, $\bar{K}_{n}^{o(\bar{m})} \in C^{3}$ and formulas (3.7) hold. Because the system of equations (3.7) is closed, we can differentiate equation (3.1) $(r-1)$ times. So we can convince ourselves that $a_{i j} \in C^{r}$, and also $\bar{g}_{i j} \in C^{r}\left(\equiv \bar{K}_{n}^{o(\bar{m})} \in C^{r}\right)$.

\section{REFERENCES}

[1] D. V. Alekseevsky, "Pseudo-Kähler and para-Kähler symmetric spaces." in Handbook of pseudoRiemannian geometry and supersymmetry. Zürich: EMS, 2010, pp. 703-729, doi: 10.4171/079$1 / 21$.

[2] H. Chudá, M. Chodorová, and M. Shiha, "On composition of conformal and holomorphically projective mappings between conformally Kählerian spaces," J. Appl. Math. Bratislava, vol. 5, no. 3, pp. 91-96, 2012.

[3] H. Chudá and M. Shiha, "Conformal holomorphically projective mappings satisfying a certain initial condition." Miskolc Math. Notes, vol. 14, no. 2, pp. 569-574, 2013.

[4] V. Domashev and J. Mikeš, "Theory of holomorphically projective mappings of Kählerian spaces." Math. Notes, vol. 23, pp. 160-163, 1978, doi: 10.1007/BF01153160.

[5] I. Hinterleitner, "On holomorphically projective mappings of $e$-Kähler manifolds." Arch. Math., Brno, vol. 48, no. 5, pp. 333-338, 2012, doi: 10.5817/am2012-5-333.

[6] I. Hinterleitner and J. Mikeš, "Geodesic mappings of (pseudo-)Riemannian manifolds preserve class of differentiability." Miskolc Math. Notes, vol. 14, no. 2, pp. 575-582, 2013. 
[7] I. Hinterleitner and J. Mikeš, "Geodesic mappings and Einstein spaces." in Geometric methods in physics. XXX workshop. Basel: Birkhäuser, 2013, pp. 331-335, doi: 10.1007/978-3-0348-0448$6 \_28$.

[8] E. Kähler, "Über eine bemerkenswerte Hermitesche Metrik," Sem. Hamburg. Univ., vol. 9, pp. 173-786, 1933, doi: 10.1007/bf02940642.

[9] J. Mikeš and all, Differential geometry of special mappings. Olomouc: Palacký University, Faculty of Science, 2015.

[10] J. Mikeš, S. Bácsó, and V. Berezovski, "Geodesic mappings of weakly Berwald spaces and Berwald spaces onto Riemannian spaces.” Int. J. Pure Appl. Math., vol. 45, no. 3, pp. 413-418, 2008.

[11] J. Mikeš, A. Vanžurová, and I. Hinterleitner, Geodesic mappings and some generalizations. Olomouc: Palacký University, Faculty of Science, 2009.

[12] Mikeš, J., "On holomorphically projective mappings of Kählerian spaces. (Russian)," Ukr. Geom. Sb., vol. 23, pp. 90-98, 1980.

[13] Mikeš, J., "Holomorphically projective mappings and their generalizations," J. Math. Sci., New York, vol. 89, pp. 1334-1353, 1998, doi: 10.1007/bf02414875.

[14] Mikeš, J. and Shiha M. and Vanžurová A., "Invariant objects by holomorhpically projective mappings of parabolically Kähler spaces.” J. Appl. Math. 2:1, pp. 135-141, 2009.

[15] T. Ötsuki and Y. Tashiro, "On curves in Kählerian spaces," Math. J. Okayama Univ., vol. 4, pp. 57-78, 1954

[16] M. Prvanović, "Holomorphically projective transformations in a locally product space," Math. Balk., vol. 1, pp. 195-213, 1971.

[17] M. Shiha, "Geodesic and holomorphically projective mappings of parabolically kählerian spaces," Ph.D. dissertation, Moscow Ped. Inst., 1992.

[18] M. Shiha, "On the theory of holomorphically-projective mappings of parabolically-Kählerian spaces," Math. Publ. 1, Silesian Univ. Opava, pp. 157-160, 1993.

[19] M. Shiha, L. Juklová, and J. Mikeš, "Holomorphically projective mappings onto Riemannian tangent-product spaces," J. Appl. Math. Bratislava, 5:3, pp. 259-266, 2012.

[20] M. Shiha and J. Mikeš, "On holomorphically projective flat parabolically-Kählerian spaces." in Proc. Conf. on Contemp. Geom. and Related Topics. Belgrade: Univ. Belgrade, Faculty of Mathematics, 2006, pp. 467-474.

[21] M. Shikha and J. Mikeš, "On equidistant, parabolically Kählerian spaces," Tr. Geom. Semin., vol. 22, pp. 97-107, 1994.

[22] P. A. Shirokov, "Selected investigations on geometry," Kazan' Univ. Press, p. 432, 1966.

[23] S.-I. Tachibana and S. Ishihara, "On infinitesimal holomorphically projective transformations in Kählerian manifolds." Tohoku Math. J. (2), vol. 12, pp. 77-101, 1960, doi: $10.2748 / \mathrm{tmj} / 1178244489$.

[24] V. Vishnevskij, A. Shirokov, and V. Shurygin, "Spaces over algebras," Kazan’: Izd. Kazansk. Univ. 263 p., 1985.

[25] K. Yano, "Differential geometry on complex and almost complex spaces," Oxford-London-New York-Paris-Frankfurt: Pergamon Press. XII, 323 p. (1965)., 1965.

\section{Authors' addresses}

\section{P. Peška}

Palacky University, Dept. of Algebra and Geometry, 17. listopadu 12, 77146 Olomouc, Czech Republic,

E-mail address: patrik_peska@seznam.cz 
J. Mikeš

Palacky University, Dept. of Algebra and Geometry, 17. listopadu 12, 77146 Olomouc, Czech Republic,

E-mail address: josef.mikes@upol.cz

H. Chudá

Thomas Bata University, Dept. of Mathematics, Nad Stráněmi 4511, 76005 Zlín, Czech Republic, E-mail address: chudalfai.utb.cz

\section{Shiha}

Al-Baath University, Dept. of Mathematics, Homs, Syria,

E-mail address: d.mohsen. sheha@gmail.com 